This item was submitted to Loughborough's Research Repository by the author.

Items in Figshare are protected by copyright, with all rights reserved, unless otherwise indicated.

\title{
A new potential for radiation studies of borosilicate glass
}

\section{PLEASE CITE THE PUBLISHED VERSION}

http://dx.doi.org/10.1016/j.nimb.2016.12.007

\section{PUBLISHER}

(C) Elsevier

\section{VERSION}

AM (Accepted Manuscript)

\section{PUBLISHER STATEMENT}

This work is made available according to the conditions of the Creative Commons Attribution-NonCommercialNoDerivatives 4.0 International (CC BY-NC-ND 4.0) licence. Full details of this licence are available at: https://creativecommons.org/licenses/by-nc-nd/4.0/

\section{LICENCE}

CC BY-NC-ND 4.0

\section{REPOSITORY RECORD}

Alharbia, Amal F., Kenny Jolley, Roger Smith, Andrew Archer, and Jamieson Christie. 2016. "A New Potential for Radiation Studies of Borosilicate Glass". figshare. https://hdl.handle.net/2134/23535. 


\title{
A new potential for radiation studies of borosilicate glass
}

\author{
Amal F. Alharbi ${ }^{1}$, Kenny Jolley ${ }^{1}$, Roger Smith $^{1}$, Andrew J. Archer ${ }^{1}$ and Jamieson K. Christie ${ }^{2}$ \\ 1. Department of Mathematical Sciences, Loughborough University, Loughborough, Leicestershire LE11 3TU, UK \\ 2. Department of Materials, Loughborough University, Loughborough, Leicestershire LE11 3TU, UK
}

\begin{abstract}
Borosilicate glass containing $70 \mathrm{~mol} \% \mathrm{SiO}_{2}$ and $30 \mathrm{~mol} \% \mathrm{~B}_{2} \mathrm{O}_{3}$ is investigated theoretically using fixed charge potentials. An existing potential parameterisation for borosilicate glass is found to give good agreement for the bond angle and bond length distributions compared to experimental values but the optimal density is $30 \%$ higher than experiment. Therefore the potential parameters are refitted to give an optimal density of $2.1 \mathrm{~g} / \mathrm{cm}^{3}$, in line with experiment. To determine the optimal density, a series of random initial structures are quenched at a rate of $5 \times 10^{12} \mathrm{~K} / \mathrm{s}$ using constant volume molecular dynamics. An average of 10 such quenches is carried out for each fixed volume. For each quenched structure, the bond angles, bond lengths, mechanical properties and melting points are determined. The new parameterisation is found to give the density, bond angles, bond lengths and Young's modulus comparable with experimental data, however, the melting points and Poisson's ratio are higher than the reported experimental values. The displacement energy thresholds are computed to be similar to those determined with the earlier parameterisation, which is lower than those for ionic crystalline materials.
\end{abstract}

Keywords: Molecular Dynamics, Borosilicate glass, Interatomic Potentials

\section{Introduction}

Borosilicate glass is an important material with numerous applications. Particular importance in the nuclear context is its use in the confinement and storage of radioactive waste. Borosilicate glasses have been the most widely used material for the immobilisation of both HLW (High-Level Wastes) and LLW (Low-Level Wastes) [1, 2]. In addition to their good chemical durability, mechanical integrity and thermal stability, borosilicate glasses are flexible with waste loadings and possess the capability of incorporating most of the waste elements. The process of incorporating HLW into borosilicate glasses is known as waste vitrification.

If HLW is incorporated into a glass, radiation damage can occur. The radioactive material has to be stored for many years, so computer simulation can be used to help predict the outcome. One way to model the radiation damage process is using classical molecular dynamics (MD). In order to study the effect of radiation on the glasses by $\mathrm{MD}$ it is necessary to have a model for the interaction potentials between the atoms in the system. Previous studies $[3,4,5]$ have proposed interatomic potentials that model some aspects of borosilicate glass but other aspects are not modelled sufficiently well, in particular the density. In the potential proposed in Ref. [3] a three-body term is included, which helps produce the correct structure but is far too strong for use in cascade studies. The predicted density using this model is $\approx 1.8 \mathrm{~g} / \mathrm{cm}^{3}$. With the potential from Ref. [4], the density corresponding to the minimum energy structure is found to be $2.7 \mathrm{~g} / \mathrm{cm}^{3}$, which is almost $30 \%$ greater than the experimental value of around $2.1 \mathrm{~g} / \mathrm{cm}^{3}$. However other aspects of the model are in good agreement with experiment. After quench- ing, the model from Ref. [5] produced glasses with a density of $3.67 \mathrm{~g} / \mathrm{cm}^{3}$ [7].

The objective of this work is therefore to modify the parameters of the potential energy used in Ref. [4] in order to develop a model that generates glass structures with a density closer to the experimentally observed value of $2.1 \mathrm{~g} / \mathrm{cm}^{3}$. We optimise the parameters using both the GULP [6] code for small systems and our own MD code for large systems, by quenching a randomly distributed set of atoms in a simulation box with periodic boundary conditions following the procedure described in Ref. [7].

\section{Interaction potentials}

The interatomic potential describes the interaction between pairs of atoms or the interaction of an atom with a group of atoms. All atoms repel one another if the distance between the centres $r=\left|\mathbf{r}_{\mathbf{i}}-\mathbf{r}_{\mathbf{j}}\right|$ becomes sufficiently small, as the inner electrons start to overlap. However, at larger distances the atoms can attract or repel one another, depending on the atomic charges. The potential must have an attractive and repulsive component if binding is to occur. There are many classes of potentials. We use the Buckingham potential to describe the interaction for large values of $r$ and the Ziegler-Biersack-Littmark (ZBL) potential [8] for small values of $r$ when the atomic cores overlap. The Buckingham potential is a pairwise additive function with no three-body terms of the form:

$$
V_{i j}(r)=A_{i j} \exp \left(-\frac{r}{\rho_{i j}}\right)+\frac{1}{4 \pi \epsilon_{0}} \frac{q_{i} q_{j}}{r}-\frac{C_{i j}}{r^{6}},
$$




\begin{tabular}{|c|c|c|l|l|l|l|l|}
\cline { 2 - 8 } \multicolumn{1}{c|}{} & $A(\mathrm{eV})$ & $C\left(\mathrm{eV} \cdot \AA^{6}\right)$ & $q_{1}(\mathrm{e})$ & $q_{2}(\mathrm{e})$ & $\rho_{i j}(\AA)$ & $\alpha_{1}(\AA)$ & $\alpha_{2}(\AA)$ \\
\hline $\mathrm{B}-\mathrm{B}$ & 121.1 & 0.0 & 1.4175 & 1.4175 & 0.49 & 0.35 & 0.65 \\
$\mathrm{Si}-\mathrm{Si}$ & 834 & 0.0 & 1.89 & 1.89 & 0.41 & 0.4 & 0.75 \\
$\mathrm{~B}-\mathrm{Si}$ & 337.7 & 0.0 & 1.89 & 1.4175 & 0.45 & 0.4 & 0.75 \\
$\mathrm{Si}-\mathrm{O}$ & 45296.72 & 46.1395 & 1.89 & -0.945 & 0.161 & 0.65 & 1.2 \\
$\mathrm{O}-\mathrm{O}$ & 9027 & 85.0321 & -0.945 & -0.945 & 0.265 & 0.19 & 1.6 \\
\hline
\end{tabular}

Table 1: Parameters for the Buckingham potential. Note that the values of $\rho_{i j}$ given above are the optimal values used largely throughout except where indicated, since in some places some of these are varied.

\begin{tabular}{|l|c|c|c|c|c|c|}
\cline { 2 - 7 } \multicolumn{1}{c|}{} & $c_{0}$ & \multicolumn{1}{c|}{$c_{1}$} & \multicolumn{1}{c|}{$c_{2}$} & \multicolumn{1}{c|}{$c_{3}$} & \multicolumn{1}{c|}{$c_{4}$} & \multicolumn{1}{c|}{$c_{5}$} \\
\hline B-B & 3.169 & 44.43 & -220.96 & 446.7 & 415.9 & 147.5 \\
Si-Si & 11.206 & -23.43 & 65.54 & -136.68 & 148.84 & 61.61 \\
B-Si & -0.22283 & 79.59 & -327.65 & 592.76 & -505.26 & 165.86 \\
Si-O & 5.49 & 21.413 & -89.7 & 138.08 & -96.49 & 24.79 \\
O-O & 9.983 & -18.06 & 27.28 & -20.53 & 6.82 & -0.79 \\
\hline
\end{tabular}

Table 2: Spline parameters in Eq. (3) used to connect the pair potential to the ZBL potential. The $c_{n}$ 's are in units of $\AA^{-n}$.

where $A_{i j}, \rho_{i j}$ and $C_{i j}$ are parameters of the model, as given in Table 1. The second Coulomb term is the interaction between ions with charges $q_{i}$ and $q_{j}$ on atoms $i$ and $j$, respectively. The charges are assumed to be fixed and $\epsilon_{0}$ is the electrical permittivity of free space. This operates in the region $r>\alpha_{2}$.

By changing the values of the parameters used in Ref. [4] and in particular the values of the parameter $\rho_{i j}$ that determines the effective diameters of the atoms, we obtain a model that more accurately describes the density of borosilicate glass. To decrease the density, we increased the parameters $\rho_{i j}$ applying the mixing rule [9]:

$$
\rho_{i j}=\frac{1}{2}\left(\rho_{i i}+\rho_{j j}\right)
$$

For values of $r<\alpha_{1}$ the standard ZBL potential is used [8]. We use an exponential spline function to connect between the Buckingham potential and the ZBL potential to give a smooth curve for the potential function and its derivatives. The spline function is defined in the region $\alpha_{1} \leq r \leq \alpha_{2}$ :

$$
S_{i j}(r)=\exp \left(c_{0}+c_{1} r+c_{2} r^{2}+c_{3} r^{3}+c_{4} r^{4}+c_{5} r^{5}\right)
$$

where $c_{0}, c_{1}, c_{2}, c_{3}, c_{4}$ and $c_{5}$ are constants. The values of these are given in Table 2, while the values of spline parameters $\alpha_{1}$ and $\alpha_{2}$ are shown in Table 1.

\section{Results}

\subsection{Optimised potential parameters}

To determine the optimum parameters given in Tables 1-2, we use two software packages, GULP [6] and an MD package developed by Loughborough University, LBOMD [10]. GULP is used first to do some quick estimates on small systems before using LBOMD on large systems for more detailed investigations. For each interatomic potential, we performed a set of quenches using constant volume MD in GULP for a range of different volumes to obtain sets of quenched glass structures at different densities.
The minimum of the curve gives the optimum density for the chosen set of potential parameters. We see from Fig.1 that the borosilicate glass with $\rho_{S i-S i}=0.41 \AA, \rho_{B-B}=0.49 \AA$ and $\rho_{B-S i}=0.45 \AA$ has a minimum energy at the experimental density of $2.1 \mathrm{~g} / \mathrm{cm}^{3}$. We also find that the borosilicate glass with $\rho_{S i-S i}=0.41 \AA, \rho_{B-B}=0.47 \AA$ and $\rho_{B-S i}=0.44 \AA$ had a minimum value at the density of $2.2 \mathrm{~g} / \mathrm{cm}^{3}$. Thus as expected, the optimum density decreases as the effective radii of the atoms increase. To obtain the curve in Fig. 1 a system contain 2520 atoms is quenched using MD after first checking smaller systems (252 atoms) in GULP.

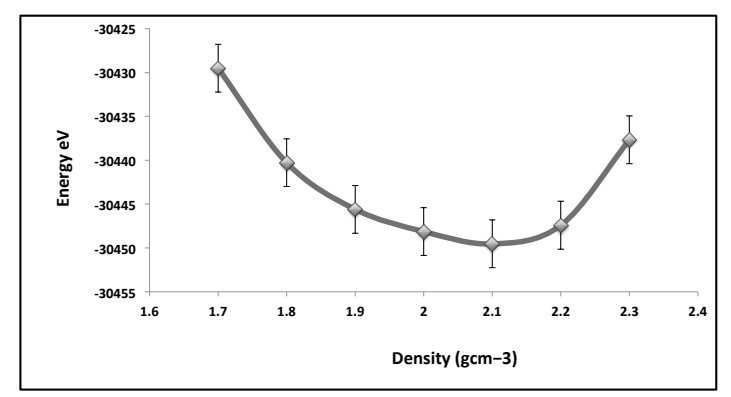

Figure 1: The energy of the glass structures plotted as a function of density using constant volume quenched MD with parameters $\rho_{S i-S i}=0.41 \AA, \rho_{B-B}=0.49$ $\AA$ and $\rho_{B-S i}=0.45 \AA$. The optimum structure has a density of $2.1 \mathrm{~g} / \mathrm{cm}^{3}$. The error bars refer to standard deviations.

\subsection{Structural properties}

Quenching borosilicate glass with $\rho_{S i-S i}=0.41 \AA, \rho_{B-B}=$ $0.49 \AA$ and $\rho_{B-S i}=0.45 \AA$ gives a glass density of $2.1 \mathrm{~g} / \mathrm{cm}^{3}$. The corresponding bond angle distributions of the borosilicate glass are shown in Figs. 2, 3, 4 and 5. The bond lengths are also in agreement with experimental data with the average B-O bond length being $1.37 \AA$, and the average for Si-O being 1.63 A.

The tetrahedral environment for Si atoms affects the shape of the O-Si-O angular distribution. In Fig.2 the O-Si-O distribution has a maximum at about $109.4^{\circ}$ corresponding to the intratetrahedral O-Si-O angle (experimentally this angle is around $109.5^{\circ}$ (Ref. [4]). The distribution for the Si-O-Si angle (Fig.3) shows a broader spread, with a maximum position consistent with the experimental result of $155.9^{\circ}$.

The local environment of boron is more complicated. The value of the $\mathrm{B}-\mathrm{O}$ distance depends on the local boron coordination number. The O-B-O angular distribution in borosilicate glass (Fig.4) indicates a peak with a maximum position consistent with the experimental results $119.7^{\circ}$ in Ref. [4]. The distribution of the B-O-B bond angle has an average at $155.6^{\circ}$ (Fig.5).

\subsection{Elastic moduli}

The calculated mechanical properties of borosilicate glass are indicated in Table 3. The elastic moduli are calculated by 


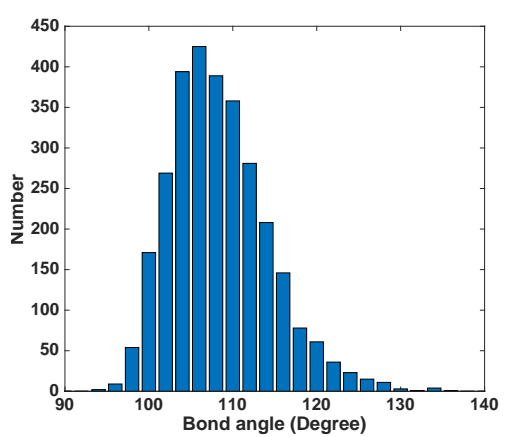

Figure 2: The distribution of the O-Si-O bond angle in borosilicate glass (average $=109 \cdot 4^{\circ}$ ).

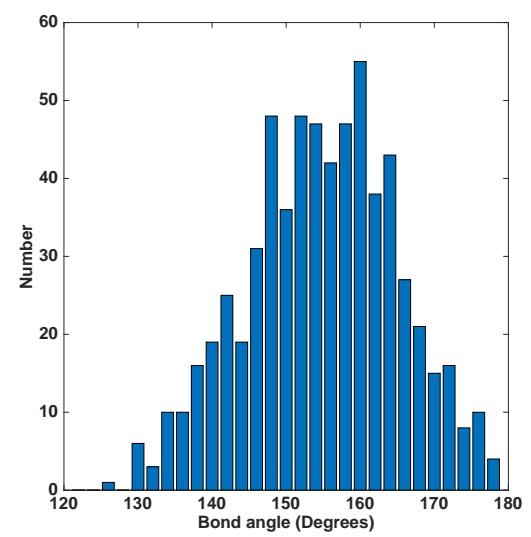

Figure 3: The distribution of the Si-O-Si bond angle in borosilicate glass (average $=155.9^{\circ}$.

GULP from the minimum energy configurations and the results shown in Table 3 are compared to the experimental values given in Ref. [4]. The values are higher than those found in experiment but show a reduction as the density of the optimal structure decreases.

\begin{tabular}{|c|c|c|}
\hline Mechanical properties & Bulk modulus (GPa) & Young's modulus (GPa) \\
\hline Calculated $\left(\rho_{B-B}=0.47 \AA\right)$ & 62.2 & 82.6 \\
Calculated $\left(\rho_{B-B}=0.49 \AA\right)$ & 57.02 & 72.72 \\
Experimental value $[4]$ & $24-49$ & $34-70$ \\
\hline
\end{tabular}

Table 3: Mechanical properties of simulated glass formulations in borosilicate glass with parameters of $\rho_{S i-S i}=0.41 \AA, \rho_{B-S i}=0.44 \AA, \rho_{B-B}=0.47 \AA$ and the structure has a density of $2.2 \mathrm{~g} / \mathrm{cm}^{3}$. The second case has $\rho_{S i-S i}=0.41 \AA$, $\rho_{B-S i}=0.45 \AA, \rho_{B-B}=0.49 \AA$ and the structure has a density of $2.1 \mathrm{~g} / \mathrm{cm}^{3}$.

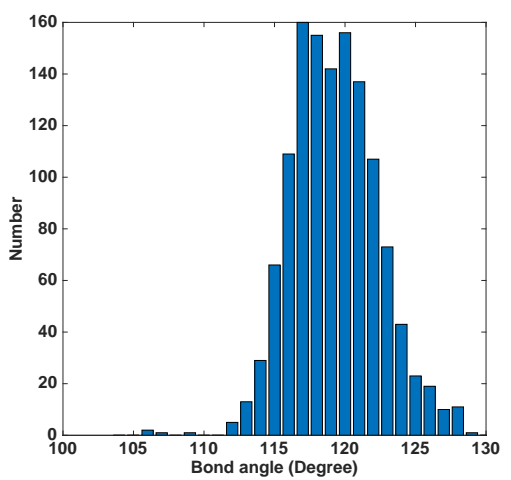

Figure 4: The distribution of the O-B-O bond angle in borosilicate glass (average $=119.8^{\circ}$ )

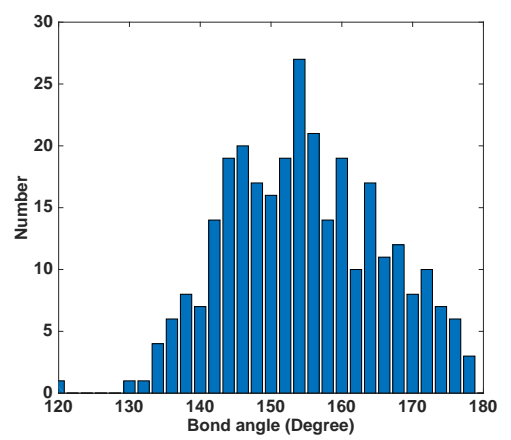

Figure 5: The distribution of the B-O-B bond angle in borosilicate glass (average $\left.=155.6^{\circ}\right)$

\subsection{Displacement energy thresholds}

The displacement energy threshold $\left(E_{D}\right)$ is an important quantity in radiation damage studies as a high value can indicate a material that is resistant to radiation whereas a low value indicates the opposite. This is not as straightforward to define as in a crystal where displacement can be identified by lattice defects.

The displacement energy threshold in an amorphous material is defined as the minimum kinetic energy that an atom needs to be permanently displaced. To do this, we randomly choose an atom from the quenched lattice and also randomly choose a direction in $3 \mathrm{D}$ space. The next step is to run an initial trial cascade by adding $20 \mathrm{eV}$ of kinetic energy to the selected atom in the random direction. Since the glass structures are amorphous, the average threshold energy does not have any significant dependence upon the initial cascade direction. After 2 ps we compare the initial and final structures to determine if the 
atom has become displaced, the criterion for this displacement is chosen as $1.2 \AA$, slightly less than the B-O bond distance of $1.37 \AA$. In the case where no displacements occur, we rerun the cascade after doubling the trial energy. The minimum threshold energy required to displace the atom is then determined using a binary search algorithm.

Figs. 6-8 show the distribution of threshold energies. Figs. 6-8 show that the $\mathrm{B}$ and $\mathrm{O}$ atoms peaks in $E_{D}$ are around 30 $\mathrm{eV}$. This rises to $40 \mathrm{eV}$ for $\mathrm{Si}$. Compared to ionic crystalline systems such as $\mathrm{MgO}$, the thresholds are lower with a more continuous spectrum see e.g. [11] but are comparable to those in $\mathrm{NaCl}$.

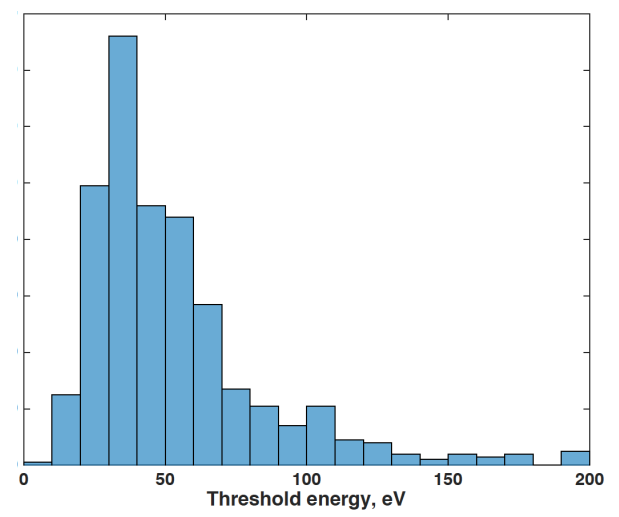

Figure 6: Histogram showing the displacement energy thresholds for $\mathrm{Si}$

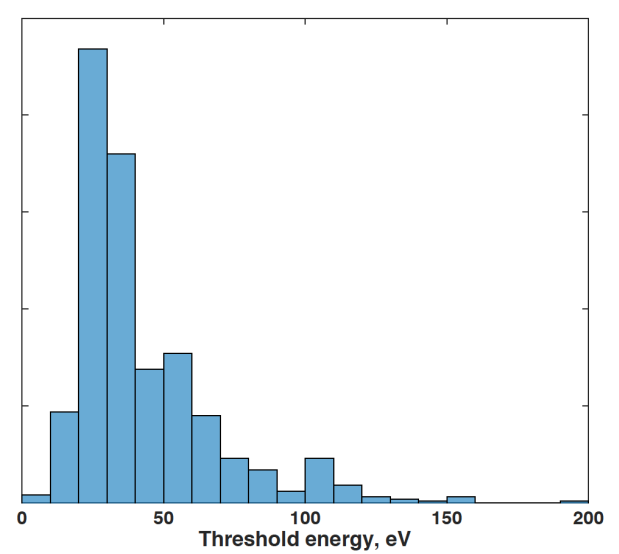

Figure 7: Histogram showing the displacement energy thresholds for B

\section{Conclusion and Future work}

We have developed a new potential for borosilicate glasses which gives a density equal to the experimental value, an improvement over previously published potentials. Structural parameters and mechanical properties are in reasonable agreement with experiment. Work is now in progress to investigate collision cascades. For crystalline materials the usual way to assess the amount of radiation damage is by counting the number of defects remaining after a collision cascade. This can

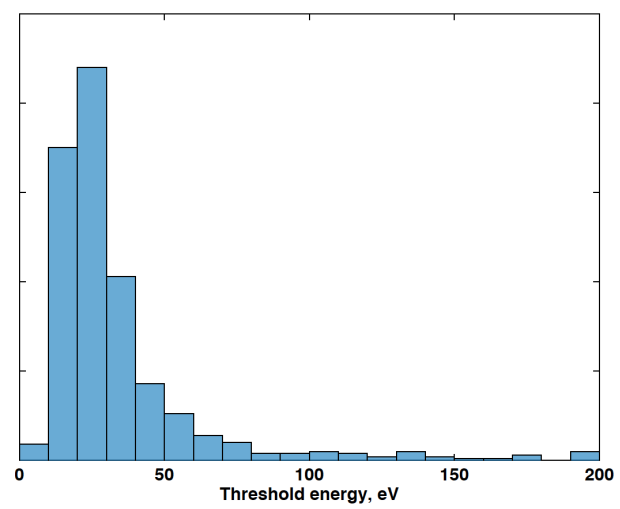

Figure 8: Histogram showing the displacement energy thresholds for $\mathrm{O}$

also be estimated using Kinchin-Pease based formulae using $E_{D}$. For glasses there is no equivalent concept of a defect. Preliminary studies indicate that the best way to classify the damage to the structure is by examining the number of displaced atoms in the entire system and the local changes in atomic co-ordination. The local structure around under and over-coordinated atoms can then be examined and energy pathways to normal co-ordination investigated. So far such pathways have been found to have high energy barriers [7] indicating that radiation-induced damage is stable over long time scales unlike point defects in crystalline atomic systems which usually can move more freely. The analysis of collision cascades and the long time evolution of the induced radiation damage will be the subject of a further study.

\section{References}

[1] W.J. Weber, R.C. Ewing. Radiation effects in glasses used for immobilization of high-level waste and plutonium disposition. J. Mater. Res. 12 (1997).

[2] J.A.C. Marples. The preparation, properties, and disposal of vitrified high level waste from nuclear fuel reprocessing. Glass Technol. 29 (1988).

[3] J.-M. Delaye, D. Ghaleb. Molecular dynamics simulation of low-energy atomic displacement cascades in a simplified nuclear glass. Journal of Nuclear Materials 244, no. 1 (1997): 22-28.

[4] L.-H. Kieu, J.-M. Delaye, L. Cormier, C. Stolz. Development of empirical potentials for sodium borosilicate glass systems. Journal of NonCrystalline Solids 357, no. 18 (2011): 3313-3321.

[5] M. Rushton, Simulations of glass and ceramic systems for nuclear waste applications (Ph.D. thesis), Imperial College, University of London, 2006.

[6] J.D. Gale, A.L. Rohl. The general utility lattice program (GULP). Molecular Simulation 29, no. 5 (2003): 291-341.

[7] K. Jolley, R. Smith, K. Joseph. Borosilicate glass potentials for radiation damage simulations. Nuclear Instruments and Methods in Physics Research Section B: Beam Interactions with Materials and Atoms 352 (2015): 140-144.

[8] J. F. Ziegler, J. P. Beirsack, and U. Littmark. The stopping and range of ions in solids, volume 1. Pergamon, New York, (1985).

[9] J.-P. Hansen, I.R. McDonald, Theory of simple liquids, Elsevier (1990).

[10] R. Smith, editor. Atomic and ion collisions in solids and at surfaces: theory, simulation and applications. Cambridge University Press, (1997).

[11] L. Kittiratanawasin, R. Smith, B. Uberuaga, K. Sickafus. Displacement threshold and Frenkel pair formation energy in ionic systems, Nucl. Instrum. Methods Phys. Res. Sect. B 268 (2010) 2901-2906. 